\title{
Truly Useful 3D Drawing System for Professional Designer by "Life-Sized and Operable" Feature and New Interaction
}

\author{
Shun'ichi Tano ${ }^{1}$, Shinya Yamamoto ${ }^{1}$, Junko Ichino ${ }^{1}$, \\ Tomonori Hashiyama ${ }^{1}$, and Mitsuru Iwata ${ }^{2}$ \\ ${ }^{1}$ University of Electro-Communications, Graduate School of Information Systems, \\ 1-5-1 Chifugaoka, Chofu-shi, Tokyo, Japan \\ ${ }^{2}$ Tokyo Metropolitan College of Industrial Technology, \\ 1-10-40 Higashioi, Shinagawa-ku, Tokyo, Japan \\ Tano@is.uec.ac.jp
}

\begin{abstract}
Media" is an artifact that expands our creativity and intelligence. We have been studying the use of "Rich Media" to support creative and intelligent human activities. Specifically, for over ten years we have focused on the 3D space as one of "Rich Media" and developed many 3D sketch systems that support the design of 3D objects. However, their long-term evaluation has revealed that they are not used by designers in real fields. Even worse, they are treated as if they were just mere attractions in an amusement park. The fundamental problem is the lack of the indispensable function for 3D space. In this paper, we propose new design principles, "life-size and operability", which make the 3D sketch system truly valuable for the designer. The new 3D sketch system is designed on the basis of "life-size and operability", developed, and evaluated successfully.
\end{abstract}

Keywords: 3D Sketch, Life-size, Operability, Professional Designer, Mixed reality.

\section{Introduction}

"Media" is an artifact that expands our creativity and intelligence. The oldest media is words and numbers. The computer is now widely used as a media.

We have been studying a wide range of creativity-centered media to ensure that systems truly support creative and intelligent human activities. They range from those used by knowledge workers to those for car-exterior designers [1-9].

Specifically, for over ten years we have developed many 3D sketch systems that support the design of 3D objects, because the 3D sketch cannot be realized without the power of advanced information communication technology (ICT) [6-9]. We regarded the $3 \mathrm{D}$ sketch as the drastic extension of the traditional "pen and paper" media made possible by the power of ICT. 
However, long-term evaluation has revealed that 3D sketches are not used by designers in real fields. Even worse, they are treated as if they were just mere attractions in an amusement park.

It shows that the rich media certainly fascinates the ordinary users but is ignored by the professional users in some cases. It may be the serious problem because there are many systems that blindly utilize the rich multimedia without the long-term user evaluation [18].

In this paper, we analyze the fundamental problems that prevent the systems from being used professionally. We point out that it is the lack of the indispensable function for 3D space. Then we propose a new design concept inspired by "mixed reality", that makes the 3D sketch system truly valuable for the designer, and exemplify the feasibility of the new design concept by describing our latest prototype system.

\section{Related Works and Purpose}

\subsection{Related Works}

Conventional research into 3D sketching can be categorized into two types. The first is generating $3 \mathrm{D}$ sketches from $2 \mathrm{D}$ sketches $[15,16]$. The designer draws a $2 \mathrm{D}$ sketch, then the system converts it into a 3D sketch on the basis of certain assumptions, and finally the system displays it in a 3D space.

The second is drawing the 3D sketch directly in midair [10-14, 17]. The 3D lines are displayed as they are or as transformed smooth lines and converted into the model description in some systems [14, 17].

We have also developed a series of 3D sketch systems [6-9] in both categories mentioned above.

Although each system has its own strength and has been successfully evaluated by the designers, the common problem is that they are not utilized continuously by professional designers in daily design tasks over long periods of time. They are missing something that would make them indispensable for professional use.

\subsection{Purpose of This Paper}

In this paper, first the role of $3 \mathrm{D}$ space is categorized into two types: "draw in $3 \mathrm{D}$ space" and "view in 3D space".

Second, our four trial systems are briefly shown as examples that support all roles of 3D space.

Third, the long-term evaluation of the four trial systems is summarized. It shows that the fundamental problem is the lack of indispensable functions for 3D space.

Fourth, we propose new design principles "life-size and operability", which make the $3 \mathrm{D}$ sketch system truly valuable for the professional designer.

Finally, our latest 3D sketch system designed by the new design principle is explained in detail and evaluated by professional designers. 


\section{Our Trials for 3D Sketch System}

We have developed a series of 3D sketch systems. The following subsection describes four typical systems [6-9] (called Systems 1, 2, 3 and 4).

\subsection{Role of 3D Space}

2D/3D space can be used in two ways as shown in Fig. 1. The first is the space where the designer draws the objects. To draw in 3D space means to draw objects in the 3D space directly, i.e., for designers to sketch them in midair in front of themselves. To draw in 2D space means to draw objects in the 2D space, i.e., using a pen and paper.

The second one is the space where the designer looks at the objects. To view in 3D space means designers look at objects in midair in front of them (stereovision). To view in $2 \mathrm{D}$ space means to look at objects as perspective $2 \mathrm{D}$ images on the $2 \mathrm{D}$ plane.

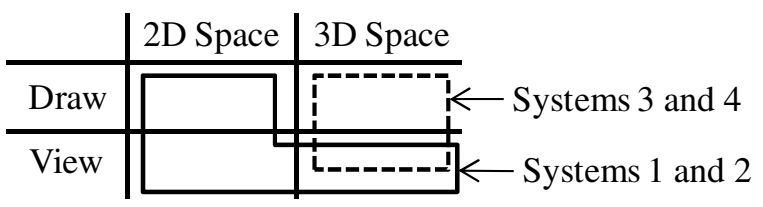

Fig. 1. "Draw in 2D/3D space" and "view in $2 \mathrm{D} / 3 \mathrm{D}$ space"

Systems 1 and 2 support the 2D-draw and 2D/3D-view. The designer draws a 2D sketch and views it both in stereovision in the 3D space and as a perspective $2 \mathrm{D}$ image on the 2D plane. The reason the systems do not support 3D-draw is the difficulty of drawing in a 3D space directly.

Systems 3 and 4 have a mechanism to compensate for this difficulty, so they support the 3D-draw and 3D-view.

\subsection{Our 3D System-1: "Godzilla"}

System 1, called "Godzilla", aims to support creative design, specifically that of car-exterior designers.

\subsubsection{Design Flow}

Fig. 2 shows the typical design flow. First, the designer draws the concept image on the 2D pad (a tablet with an LCD) as shown in Fig. 3(a). The designer can grasp the sketch and hold it in midair, and it appears as a 3D image on the 3D pad (stereovision TV) as shown in Fig. 3(b). While holding and rotating the 3D-image, the designer can look at it from different viewpoints. When the designer grasps the image and puts it onto the 2D pad, it appears on the 2D pad as a 2D sketch. Note that our system displays a hand-drawn sketch all the time, even in the 3D space, and can automatically recognize the $3 \mathrm{D}$ shape of a $2 \mathrm{D}$ image and transform between the $2 \mathrm{D}$ and $3 \mathrm{D}$ sketches with different viewpoints while preserving the designer's pen touch. 
Direct operation by pen

Direct operation by pen and gesture
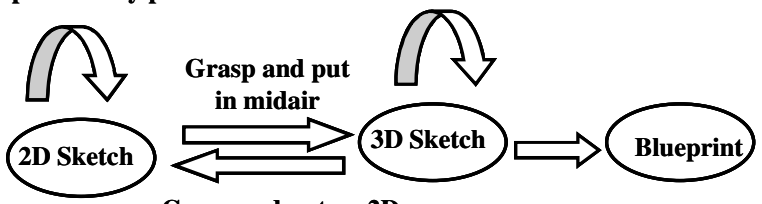

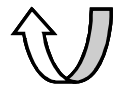

Automatic transformation by voice and pen
Grasp and put on 2D

pad

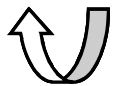

Automatic transformation by voice, pen and gesture

Fig. 2. Typical design flow

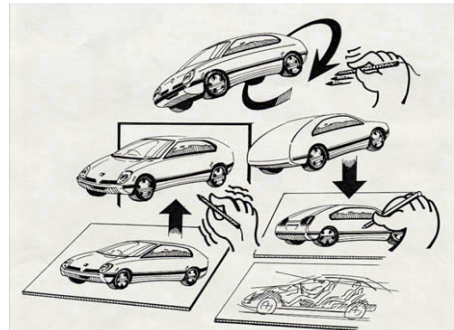

(a) Design Flow

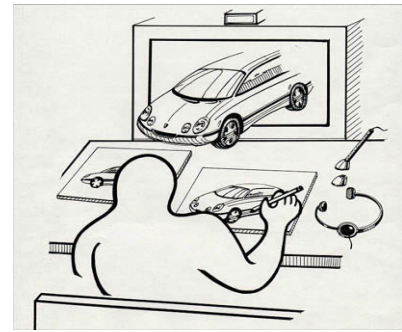

(b) Design Environment

Fig. 3. Image of design flow and environment

\subsubsection{Prototype System}

Fig. 4 is a photo of the Godzilla system. Fig. 5 shows examples of car design using Godzilla. Note that a 3D image is displayed in midair just in front of the 3D-pad. After we developed the Godzilla system, we took it to the design division of the Toyota automobile company for the initial evaluation.

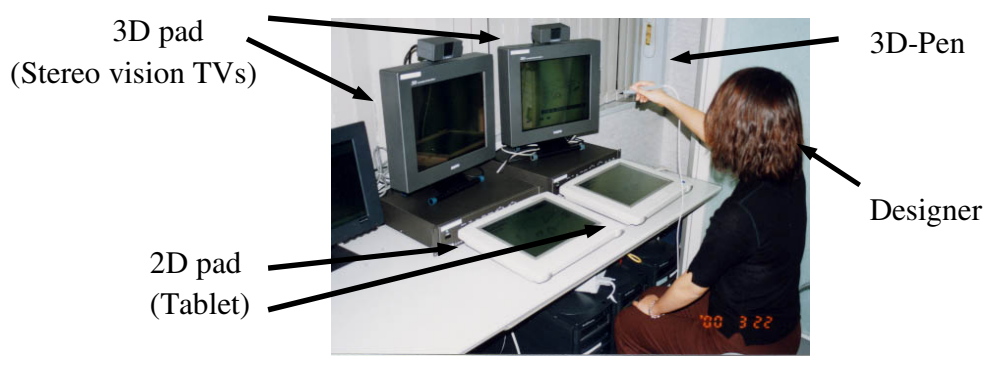

Fig. 4. Prototype system "Godzilla" 

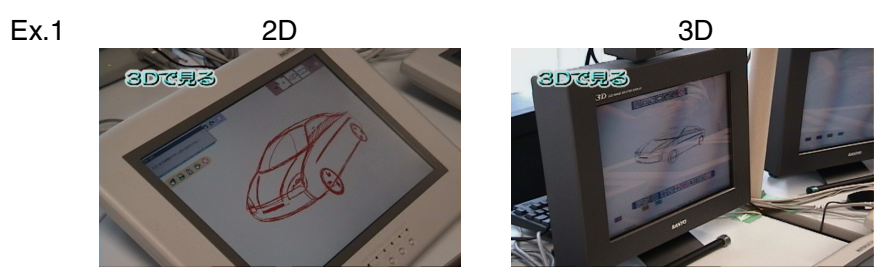

Fig. 5. Examples

\subsection{Our 3D System-2: "Extended Godzilla"}

Godzilla has problems: (1) The range of forms it can handle is restricted to car-like forms, and (2) separate 2D and 3D monitors feel unnatural.

\subsubsection{Free Form Design Using a Combination of Seven Primitives}

It is obviously impossible to recognize a $2 \mathrm{D}$ sketch of a $3 \mathrm{D}$ form without any knowledge of the sketched form since a $2 \mathrm{D}$ sketch cannot retain all of the shape information. To enable free-form design, we developed a design approach that enables the designer to draw primitive forms and then combine and modify them.

Our system has seven primitives: triangular pyramid, square pyramid, triangular prism, square prism, cone, cylinder, and sphere. A typical design flow is shown in Fig. 6. First, the designer sketches the primitive shapes, and the system recognizes them. The designer then combines and modifies the primitives or views and checks the shapes in the $3 \mathrm{D}$ space.

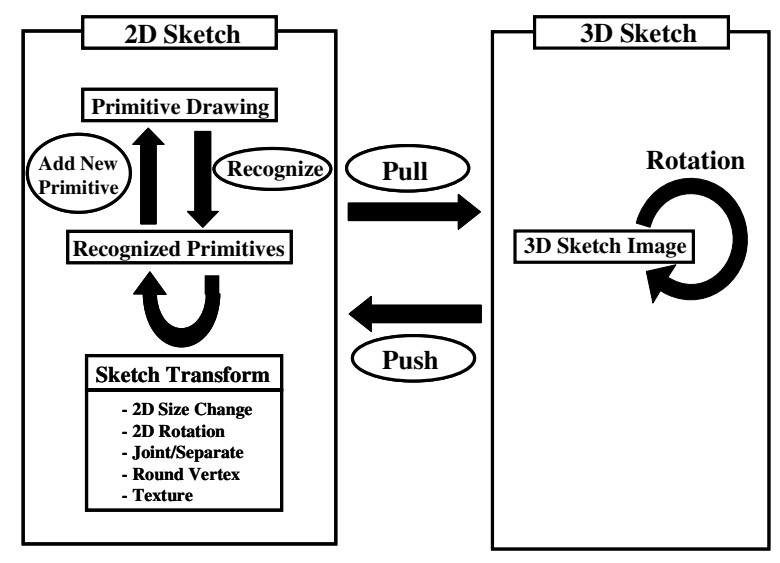

Fig. 6. Design flow in "Extended Godzilla" system

\subsubsection{Natural Seamless 2D-3D Display}

To provide a natural display, the display unit has to support the seamless 2D-3D transition. To meet these requirements, we used an LCD monitor with polarized light screens (a "micro-pole filter") and polarized glass (see Fig. 7). 


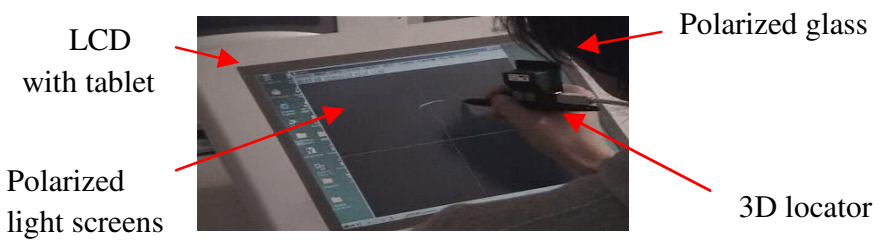

Fig. 7. "Extended Godzilla" System

The only operation in 2D-3D space is "pull and push." As shown in Fig. 8, when the designer "pulls" an image in the 2D space, the image is gradually raised from the surface of the LCD. Conversely, when the designer "pushes" an image in the 3D space, the image gradually sinks into the LCD.

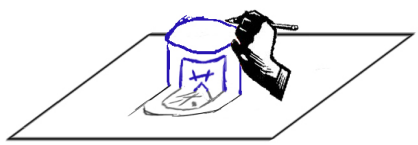

Fig. 8. "Pull and Push"

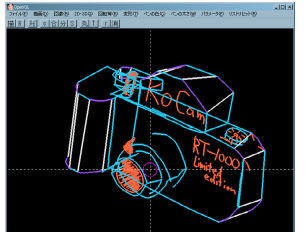

Fig. 9. Example

Representative sketches are shown in Fig. 9. Note that the images can be viewed from any angle since the sketches have a 3D structure.

\subsection{Our 3D System 3: Rich Visual Feedback}

Sketching in 3D space is difficult, particularly because the senses of depth and balance are poor. This indicates that a user interface is needed to compensate for the difficulties.

For the compensation, the interface needs to enhance the user's awareness of errors.

We found that using the metaphors of "shadow" and "hand mirror" effectively achieves this, as illustrated in Fig. 10. Note that the shadow and mirror are also 3D images.

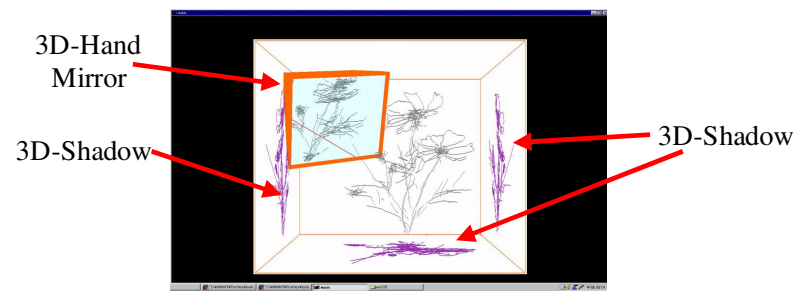

Fig. 10. Use of "Shadow" and "Hand Mirror" 


\subsection{Our 3D System-4: Force Feedback}

System 4 tried to solve the same problem as System 3 by providing the designer with the virtual force field (virtual surface) in midair.

\subsubsection{Approach}

We devised four types of force field to cope with the problem. They are characterized in Fig. 11.

(a) Uniform force field: The uniform inertia is produced in the drawing area. The designers feel as if they were drawing in jelly.

(b) Automatic generation of surface: The artificial surface is automatically generated in accordance with the drawing. When many lines are judged on the same plain, the force field is automatically generated to shape the plain.

(c) User-defined surface: The user can indicate the needed surface by gesturing.

(d) Use of 3D rulers: We designed several virtual 3D rulers, which include the virtual sphere, the virtual rod with ditch (for drawing the straight line in 3D), the virtual 3D French curve, and so on. They are virtual in the sense that they are not real objects. The surface is artificially generated at the position of $3 \mathrm{D}$ sensor, so they are easily fixed in 3D.

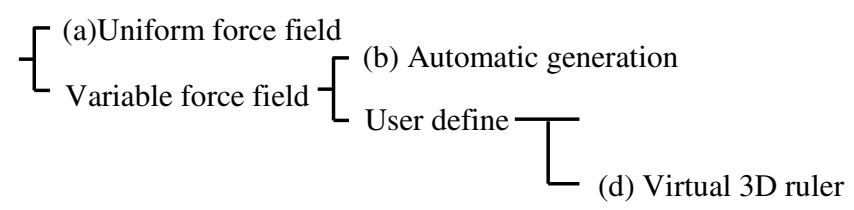

Fig. 11. Categories of four force fields

\subsubsection{Prototype System}

We have developed a prototype system (Fig. 12). The user has a 3D-sensor (for a virtual 3D-ruler) in his/her left hand and an arm of the Phantom (the force feedback device) in his/her right hand for drawing in 3D. Fig. 13 shows the automatic generation of the surface. Note that it is seen in double since the left-eye and right-eye images are displayed.

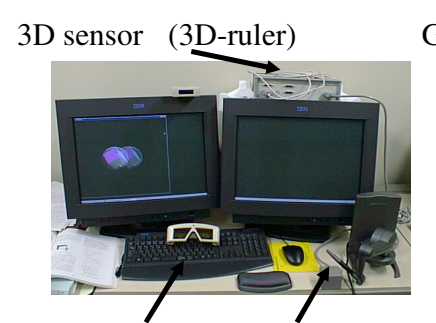

CrystalEys Arm of Phantom (3D-pen)

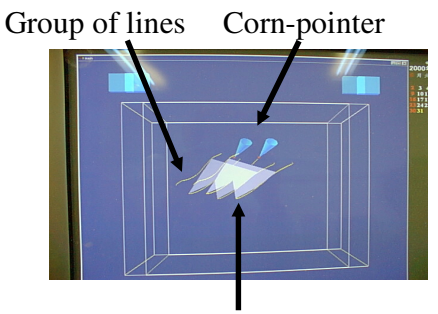

Generated virtual surface

Fig. 12. Photo of prototype system

Fig. 13. User interface 


\section{$4 \quad$ Long-Term Evaluation}

\subsection{Short-Term Evaluation Was Promising}

For each system, we conducted a short-term user test that showed our user interface was promising. All designers welcomed the novel interaction.

\subsection{Long-Term Evaluation: 3D Space Is Useless}

However, they stop using the 3D space after a while. In the following subsection, the reason for this is analyzed in terms of "view in 3D" and "draw in 3D".

\subsubsection{Analysis on "View in 3D"}

While using Godzilla (System 1), the users draw a 2D sketch and lift it up into midair. Then the 3D sketch is displayed, fascinating the users. Sometimes the user is surprised at the function. Nevertheless, sooner or later, they notice that they do not need to look at it in the 3D space as a 3D sketch. They can look at it on the 2D-pad and can rotate it in a similar manner. The difference is whether they are displayed in 3D (stereoscopic) or in semi-3D on the $2 \mathrm{D}$ plane (perspective $2 \mathrm{D}$ image).

The same phenomena were also found while using Extended Godzilla (System 2). The users draw a 2D sketch and lift it up into midair gradually. Because the sketch seamlessly transitions from $2 \mathrm{D}$ to $3 \mathrm{D}$, it surprises and fascinates all users. However, after a while, they bore of the seamless 2D-3D transition.

\subsubsection{Analysis on Drawing in 3D}

While using System 3, the designer can draw a beautiful 3D flower image directly as shown in Fig. 10 by utilizing the effect of visual feedback. Also, while using System 4 , a designer can draw a image stably by utilizing the effect of the virtual surface in midair.

However, even though the designer is assisted by the visual feedback and the force feedback, 2D drawing is much easier than 3D drawing. Therefore, the designers tend to move to System 2 ("Extended Godzilla") as long as System 2 can handle the target image, so eventually they use $2 \mathrm{D}$ draw and $2 \mathrm{D}$ view as mentioned above.

\subsection{Lack of Indispensability of 3D Space}

Essentially, the 3D space is not for a designer in terms of either "view in 3D" and "draw in 3D".

The reason designers stop using the $3 \mathrm{D}$ space is that they can do their work without it. In other words, our systems do not provide the designers with an indispensable function that truly needs 3D space. 


\section{$5 \quad$ New Design Concept}

As long as an indispensable function of 3D space is not found, it is pointless to develop a support system that uses 3D space. Therefore, we stopped our research into 3D space for a few years. Recently, we found indispensable functions and restarted the research activity. Here, the latest findings and the current prototype system are shown.

\subsection{Indispensable Functions of 3D Space}

We found two indispensable functions that need the 3D space. The first one is a lifesized 3D sketch. If the 3D sketch is life-sized, the user evaluates the size to compare their own body and the 3D sketch shown in the midair in front of the user.

If the "life-sized" nature is missing, the users cannot evaluate it on the basis of comparison with their body, so the necessity of 3D sketch is lost.

The second one is a 3D sketch that must be operable by the user. The user should be able to operate the 3D sketch, that is, touch, push, move, and so on. If the 3D sketch is operable, the user evaluates the ease of use by operating while stooping down, extending a hand, or twisting his/her body.

\subsection{Design Concept "Life-Sized and Operable"}

The 3D sketches must have a "life-sized and operable" nature. Since the life-sized 3D sketch can be evaluated by comparison with a user's body, it needs to be displayed in a 3D space in front of the user. For example, the user can notice that the table of this kitchen is low or the emergency button is far from the operator's chair.

Similarly, since users can evaluate the operational 3D sketch by moving their bodies while operating, it needs to be displayed in a 3D space in front of the users. For example, the user can notice when operating a lever that the warning lamp is hard to see or the tray of the copy machine is too low to remove the paper jam.

\section{New 3D Sketch System with Mixed Reality}

\subsection{New Design Flow}

Fig. 14 shows the new design process extended by the new principle. The design flow is explained briefly by using the copy machine design example (see also Fig. 15).

In the first step, the designer is thinking of a shape of a copy machine, asking him or herself, "What's a smart design for a copy machine?" while drawing the idea in life-size and ubiquitously. 


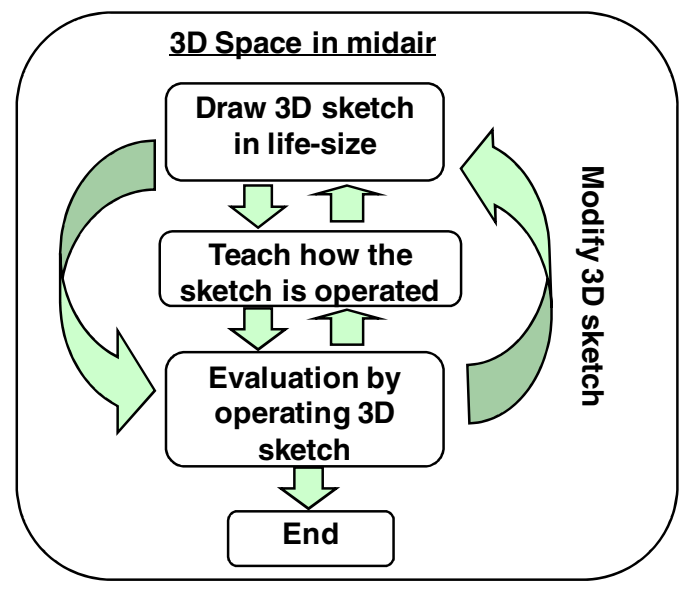

Fig. 14. New design process

Second, the designer is thinking of the operation of the sketch, thinking to him or herself, "The tray will move in this direction. If I push this button, the paper is ejected.", then formulating the operation rules by grasping and moving the sketch shown in 3D.

Third, the designer checks the usability by operating the sketch while sitting down, stooping down, extending an arm, and so on.

Then the designer may find that a button is hard to push because it is inconveniently located, the tray is hard to pull out because you have to get into an uncomfortable position, and so on. The designer simply erases the 3D sketch and redraws it.

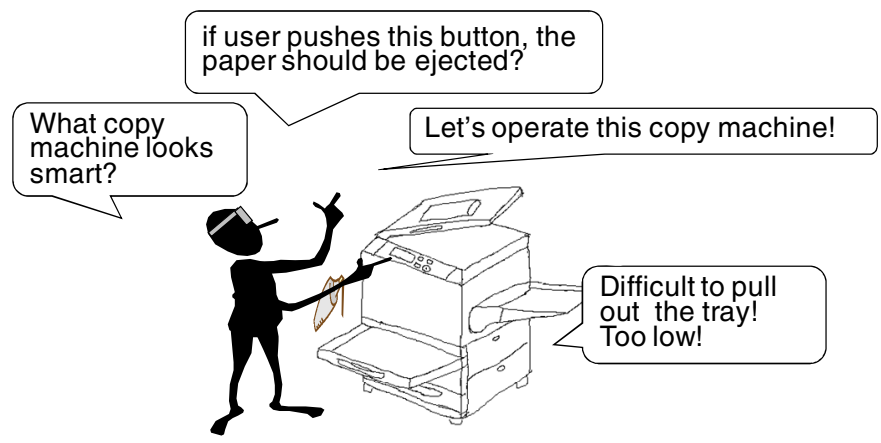

Fig. 15. Copy machine design example

\subsection{Examples of Design Process}

Fig. 16 shows our other application field, i.e., the control room design. It is very important to design a usable control room from the safety point of view. It is impossible to make a mock-up system due to the exorbitant costs. In a real design department, the designers will design it virtually by using the 3D-CAD system. During the design process, the designers cannot fully appreciate the size and operability. All they can do is to imagine the size and the operation scene in their mind. 
By using our latest prototype system, the designer starts to draw the control room roughly (Fig.16 (a)). Then the designer teaches the system the operation of levers, buttons, and warning lights (Fig.16 (b)). The designer finds several problems (Fig.16 (c)) and fixes them by redrawing the sketch (Fig.16 (d)).

Note that all this is done by hand-drawn sketches. The scenario can be done in less than one hour.

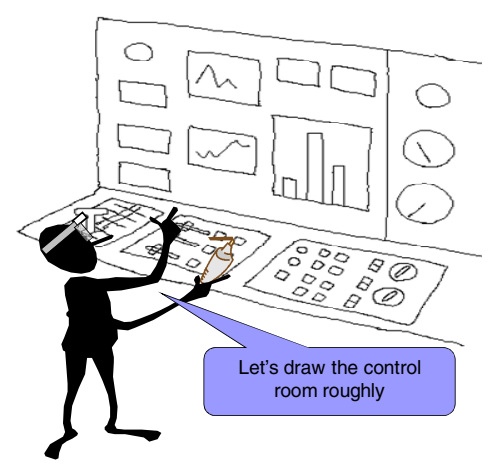

(a) Initial Sketch

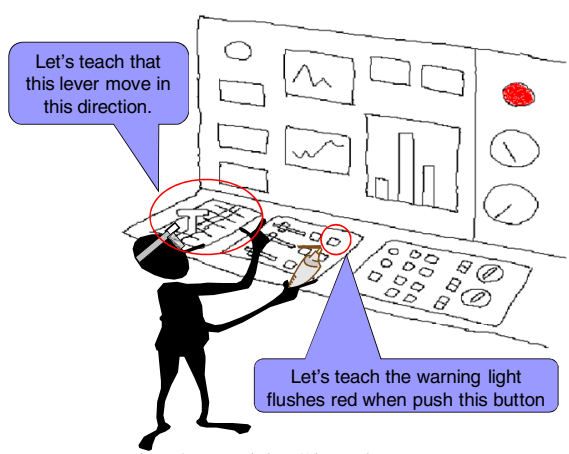

(b) Operable Sketch

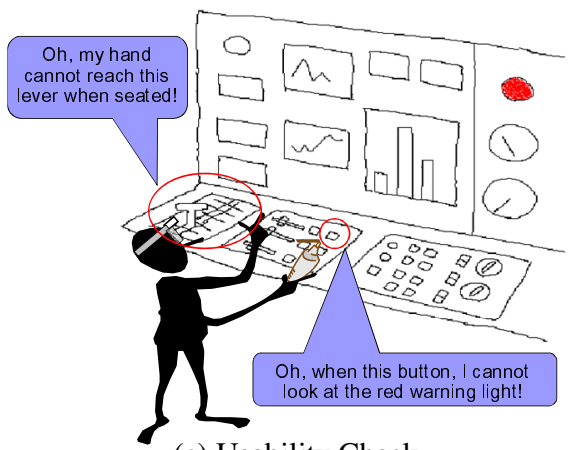

(c) Usability Check

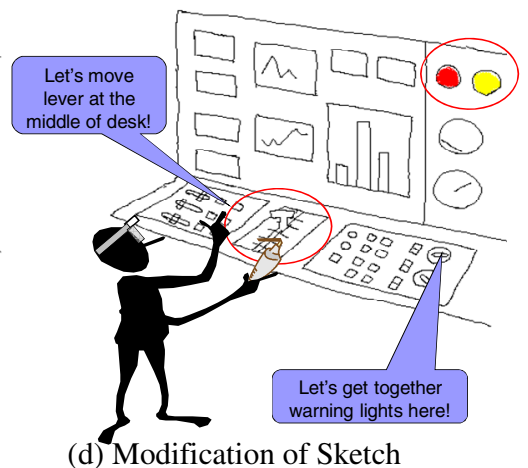

(d) Modification of Sketch

Fig. 16. Control room design example

\subsection{Special User Interaction for the New Design Concept}

The new design concept "life-size and operability" requires new interaction methods.

\subsubsection{Interactions for "Life-Size"}

Since it is difficult to draw a life-size image, our system has several unique interactions. Here three interactions are shown.

The color of the drawing line changes at the fixed length $(50 \mathrm{~cm})$ as shown Fig. 17. The designer can recognize the length of the line while drawing the lines. 


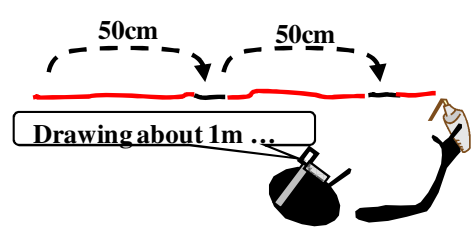

Fig. 17. Line length notification

Fig. 18 shows the lead line. It is a kind of auxiliary line. The designer is drawing a refrigerator (red box) between two real drawers. The lead line-A connects the designer's body (left hand) and the virtual image (refrigerator: red box). If the designer moves his/her left hand, the lead line-A moves too. The lead line-B connects two real objects. It is literally a static auxiliary line drawn in the real world. The lead line-C connects the designer's body (waist) and the real object (right drawer).

These lead lines help the designer grasp the 3D location of real and virtual objects.

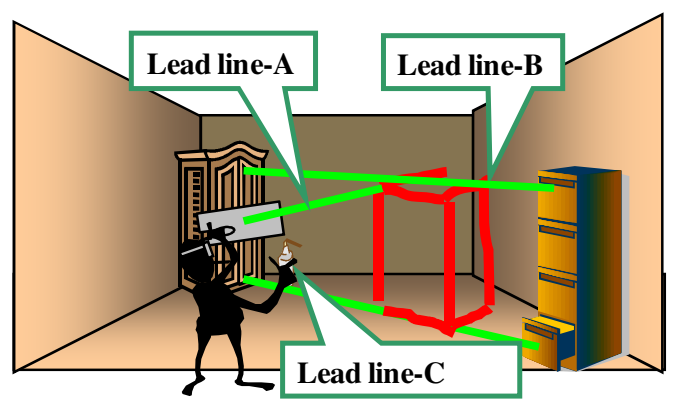

Fig. 18. Three types of lead line

Since the drawings are life-sized, they tend to be big. Therefore, the selection of the object is difficult. Our system has a few selection methods. Fig. 19 shows the projection based selection. The designer draw a closed line in front of him/her, the closed line is projected conically from the center of the designer's eye. The drawings included in the projected volume are selected. The closed line can be drawn in the different space. Then the merged volume is the selected zone as shown Fig. 19.

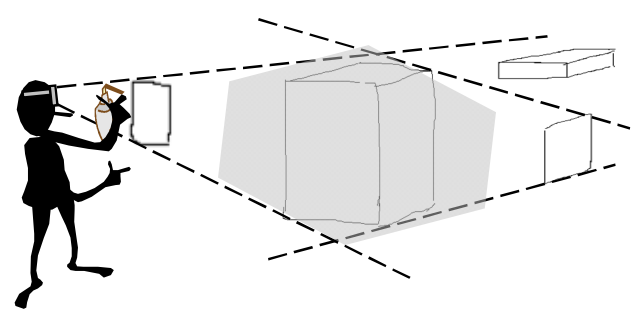

Fig. 19. Selection by projection 


\subsubsection{Interactions for "Operability"}

The designer teaches the part of sketch how to respond to the users' operation (behavior) in three steps. An example (Fig.20) shows how to teach the operability of the control board, which consists of two color lights and one lever. The operability of the control board as follows;

- Lever moves vertically

- Light changes color in accordance with the lever's position

In the first step, the designer draws the overview of the control panel with two color lights and one lever (Fig.20 (a)). In the second step, the designer teaches the operation of single component, i.e., the state transition (ex. On/OFF switch, color light) and the trajectory movement (ex. lever (vertical move), dial (cursive move)). For example, in Fig.20 (b), the designer teaches the trajectory movement of the lever by selecting the sketch of lever and moving it vertically. In the last step, the designer teaches the cooperation of multiple components. In Fig.20 (c), the designer teaches the cooperation of the lever and the light by moving the lever at the terminal point and then switching the light to the preferred color.

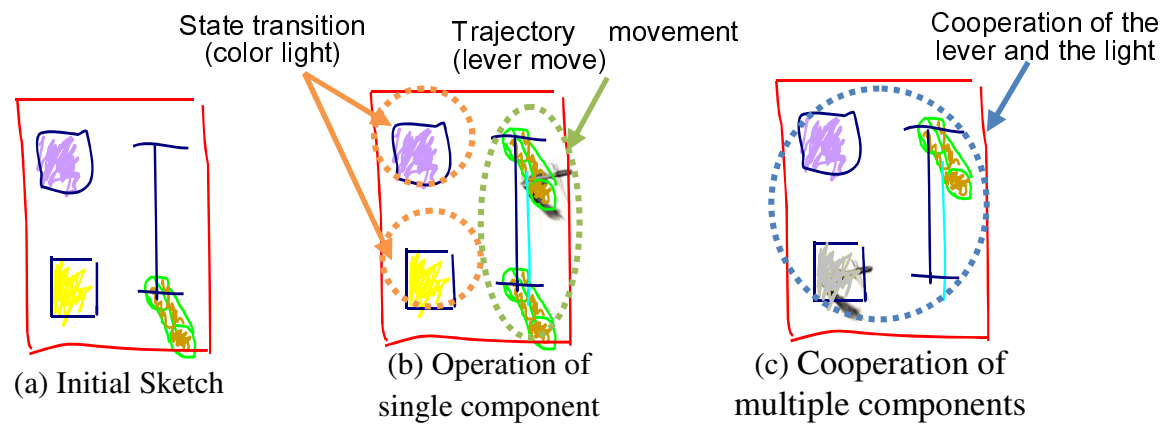

Fig. 20. New interactions for "operability"

\subsection{Current Prototype System}

The current prototype system consists of a see-through HMD, head-tracker, 3D-pen, and palette (command board) as shown in Fig. 21. The 3D position sense is implemented by combining the ultrasonic and magnetic sensor to handle a large 3D sketch, such as a control room. To promote the cooperative design, three or more HMDs (maximum 6) are connected.

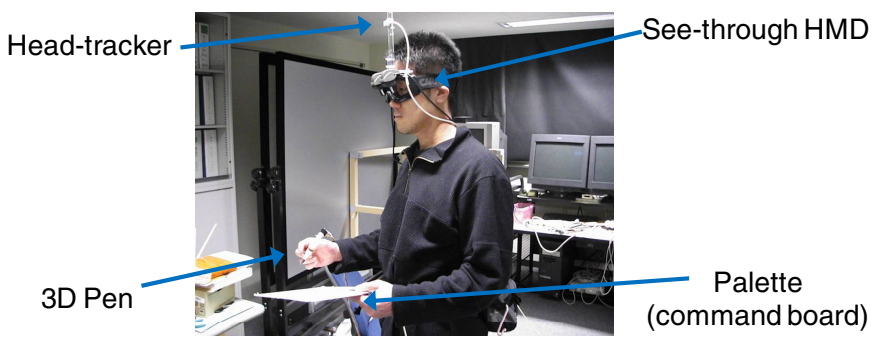

Fig. 21. Current prototype 
The prototype system has $3 \mathrm{~m} \times 4 \mathrm{~m}$ open space as the cooperative design space (Fig.22 (a)). Two types of 3D position sensor are equipped on the ceiling (ultrasonic sensor) and the edge of the wall (magnetic sensor) to provide the users with the spacious open area.

The current prototype system is implemented by single PC architecture shown in Fig.22 (b). The main PC has two graphic boards (Quadro 4000) which have $2400 \mathrm{x}$ 600 dot screen each. The $2400 \times 600$ dot screen is divided into $3800 \times 600$ dot screens by Matrox TripleHead2GO which are connected to the see-through 3D-HMDs (eMagin Z800). This simple architecture realized 6 users environment by the single computer.
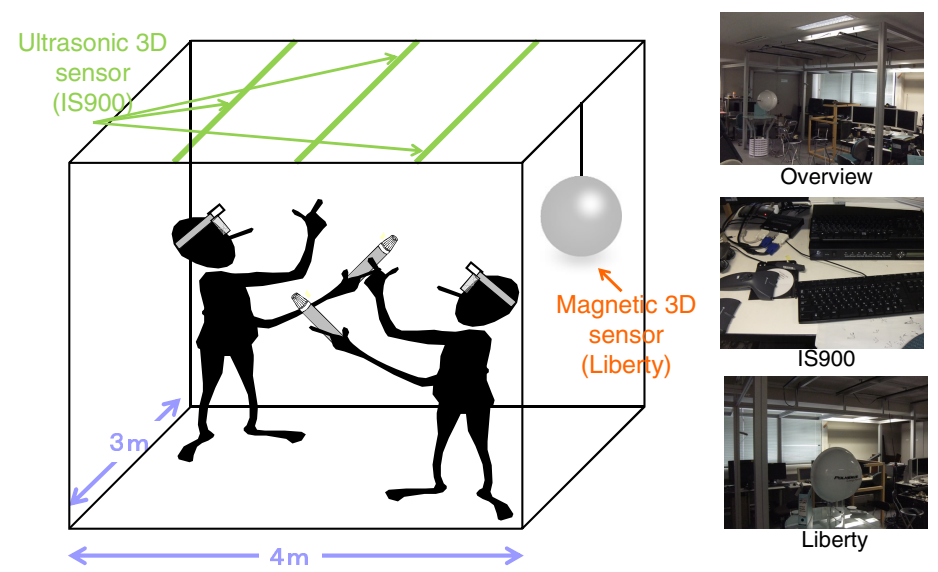

(a)Design Space (6 designers, $3 \mathrm{~m} \times \mathrm{m}$ )

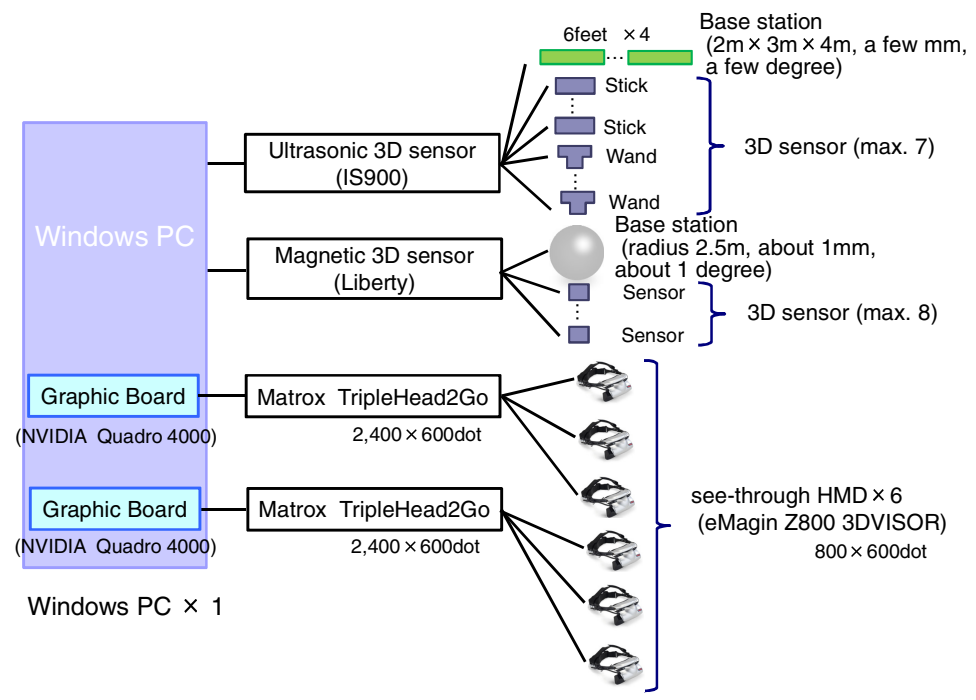

(b) System Architecture

Fig. 22. System overview 
Fig. 23 shows examples of what the users view through the HMD. As shown in Fig. 23(a), a virtual pen is displayed on the user's real world pen. Fig. 23 (b) shows an example in which the designer draws a 3D sketch by referring to a real object's size.

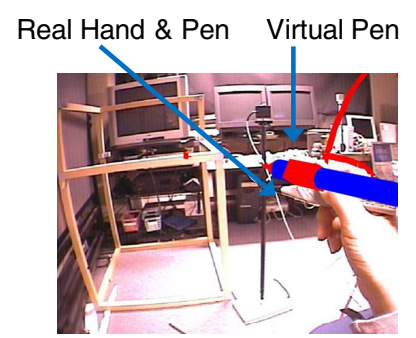

(a) Virtual Pen

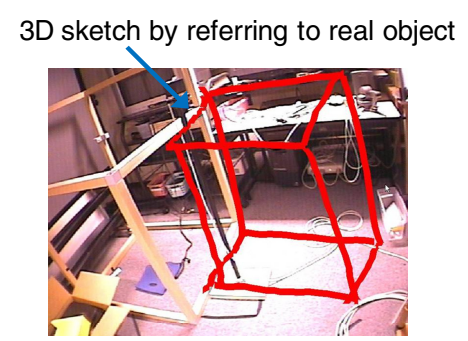

(b) Object Drawing

Fig. 23. User's view through HMD

\subsection{Preliminary Evaluation}

We conducted a preliminary evaluation with the help of professional designers. Fig. 24 and Fig. 25 are the photos of the experiment.
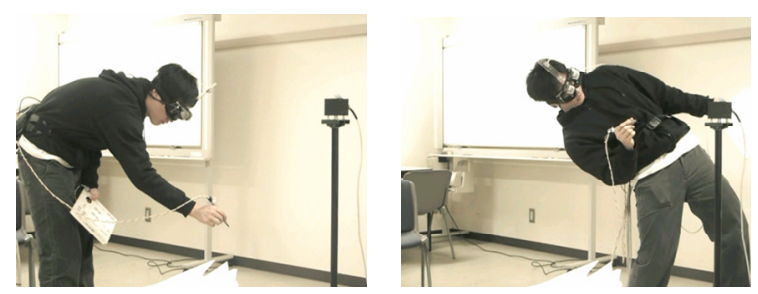

Fig. 24. Behavior of subject-A
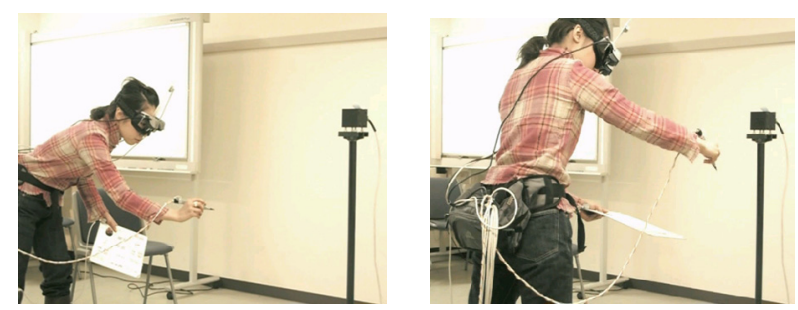

Fig. 25. Behavior of professional designer

The subjects were 5 students and 1 professional designer. All of the subjects took a training course that covered all interaction methods in 17 scenarios. The preliminary evaluation took place after they completed all the scenarios.

In the preliminary evaluation, all subject were given the design theme concerning to the new copy machine, then they began to design freely. 
We videotaped the design process of 6 subjects and analyzed the specific behaviors.

Table 1 shows the frequency of the new interactions for "life-size", proposed in the section 6.3.1. All subjects have used all interactions and frequently used the selection by projection. The prototype system had several methods for the selection since it was difficult to select the part of the sketch that floated in the midair. For example, the prototype system had the direct selection method in which the user touched one line by one line and the touched lines were selected, the volumetric selection method in which the user defined the rectangular solid by drawing the diagonal line, and the lines in the volume were selected, and so on. Because of the "life size" nature, the selection by projection seemed to be preferred.

Table 1. Behavior of "life-size" interaction

\begin{tabular}{|c|c|c|c|c|}
\hline & $\begin{array}{c}\text { \# of Line length } \\
\text { notification }\end{array}$ & \# of Lead line & $\begin{array}{c}\text { \# of Selection by } \\
\text { projection }\end{array}$ & $\begin{array}{c}\text { \# of Volumetric } \\
\text { selection }\end{array}$ \\
\hline Subject-A & 6 & 2 & 9 & 5 \\
\hline Subject-B & 4 & 2 & 4 & 7 \\
\hline Subject-C & 1 & 2 & 7 & 3 \\
\hline Subject-D & 6 & 3 & 6 & 4 \\
\hline Subject-E & 7 & 1 & 12 & 6 \\
\hline Designer & 4 & 2 & 7 & 1 \\
\hline Average & 4.7 & 2 & 7.5 & 4.3 \\
\hline
\end{tabular}

Table 2 shows the behavior of the "operable" interaction. "\# of operable sketch" does not mean the number of the operable sketches which remained in the final design but the number of the operable sketches which were drawn in all the design process (i.e., includes the try and error sketches). All subjects drew many operable sketches. Since one co-operable sketch consists of at least two single component, so the average number 5.5 means that at least 11 components are used in the co-operation. Surprisingly, almost all single components worked with other components. Also the number of operations 16.0 was so high that they seemed to focus on the usability evaluation.

Table 2. Behavior of "operable" interaction

\begin{tabular}{|c|c|c|c|}
\hline & $\begin{array}{c}\text { \# of Operable sketch } \\
\text { (single component) }\end{array}$ & $\begin{array}{c}\text { \# of Co-operable } \\
\text { sketch (multiple } \\
\text { components) }\end{array}$ & $\begin{array}{c}\text { \# of User operation } \\
\text { (behavior) }\end{array}$ \\
\hline Subject-A & 16 & 7 & 13 \\
\hline Subject-B & 8 & 2 & 12 \\
\hline Subject-C & 10 & 4 & 9 \\
\hline Subject-D & 12 & 8 & 15 \\
\hline Subject-E & 22 & 6 & 21 \\
\hline Designer & 11 & 6 & 26 \\
\hline Average & 13.1 & 5.5 & 16 \\
\hline
\end{tabular}


Table 3 lists several statistics on the final design. They tend to use many colors that were used to show the different parts. The final design contained 6.8 operable components and 2.5 co-operable rules in average. They drew more operable sketch than we expected. Only half of the sketch components remained in the final design. It might be explained by the trial and error approach in designing of the interaction.

Table 3. Statistics of final design

\begin{tabular}{|c|c|c|c|}
\hline & \# of Colors & $\begin{array}{c}\text { \# of Operable sketch } \\
\text { (single component) }\end{array}$ & $\begin{array}{c}\text { \# of Co-operable } \\
\text { sketch (multiple } \\
\text { components) }\end{array}$ \\
\hline Subject-A & 7 & 10 & 3 \\
\hline Subject-B & 6 & 6 & 2 \\
\hline Subject-C & 6 & 4 & 2 \\
\hline Subject-D & 11 & 9 & 4 \\
\hline Subject-E & 7 & 6 & 3 \\
\hline Designer & 8 & 6 & 3 \\
\hline Average & 7.5 & 6.8 & 2.5 \\
\hline
\end{tabular}

Fig. 26 and Fig. 27 are the results of the design. Note that the designs are real-sized and operable. Fig. 27 is the design result when the designer was asked to design a novel copy machine such as "never before seen copy machine". They successfully designed a "novel operable" copy machine, which was a round copy machine usable from any direction. Note that the round tray is also operable.

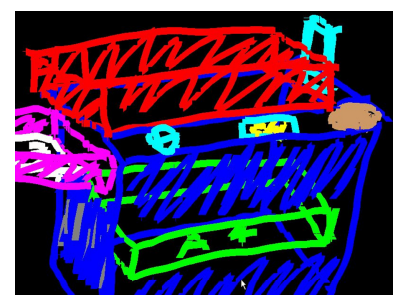

(a) Overview

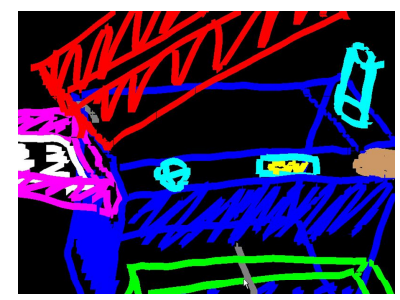

(b) Operation of Upper Tray

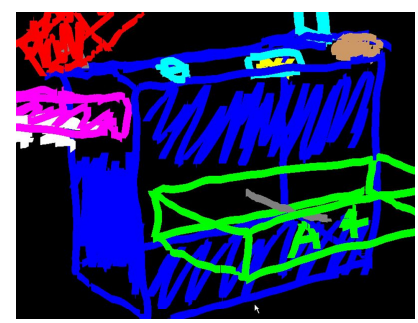

(c) Operation of Paper Tray

Fig. 26. Design result-A 


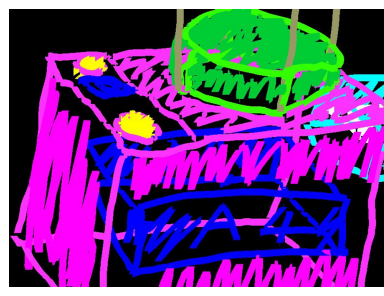

(a) Overview

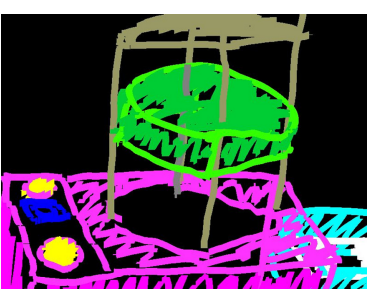

(b) Operation of Upper Tray

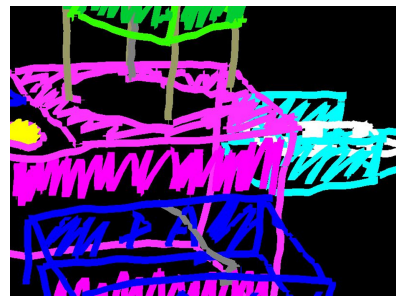

(c) Operation of Paper Tray

Fig. 27. Design result-B

Fig. 28 is the analysis of the professional designer's behavior while operating the 3D sketch. Fig. 28 implies that the designer willingly drew the life-seized sketch and frequently operated the 3D sketch to check the usability of the copy machine.

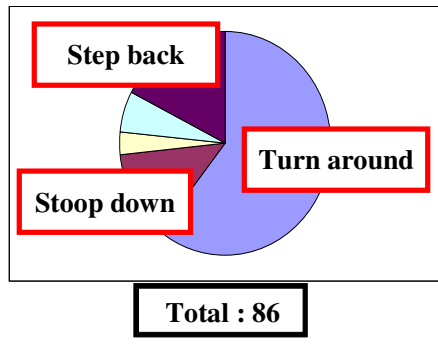

(a) Analysis-A

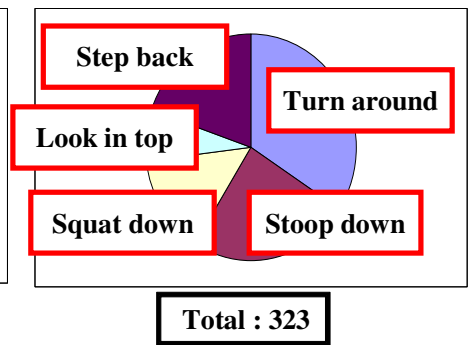

(b) Analysis-B

Fig. 28. Analysis of professional designer's behavior

\section{Conclusion}

This research began from the serious realization that 3D sketch systems were not used by professional designer in real design fields. They are treated as if they were just mere attractions in an amusement park.

In this paper, we proposed a new design concept, "life-size and operability", which should make the 3D sketch system truly valuable for the designer.

According to the preliminary evaluation, the design concept seems promising. However, its validity has not yet been completely proven. We are now obtaining the data by applying it to real world design. 
We hope our research is helpful to design the multimedia-based support system for professional use.

\section{References}

1. Dzulkhiflee, M., Tano, S., Iwata, M., Hashiyama, T.: Effectiveness of Annotating by Hand for non-Alphabetical Languages. In: CHI 2006, pp. 841-850 (2006)

2. Iwata, M., Sasaki, Y., Tano, S., Hashiyama, T., Ichino, J.: A Sketch Support System Based on Behavior of Designers. In: IEEE International Conference on Progress in Informatics and Computing-2010, pp. 1298-1304 (2010)

3. Ichino, J., Makita, T., Tano, S., Hashiyama, T.: Support for seamless linkage between lessdetailed and more-detailed representations for comic design. In: CHI 2009, pp. 3979-3984 (2009)

4. Tano, Kamura, Iwata, Hashiyama: Digital Paper Concept for Reflective Writing by Seamless Traverse between Handwritten and Coded Information. In: HCI International 2005, E-book (2005)

5. Tano, S.: Quantitative Study on the Effectiveness of Pen-based Computing on Experiential and Reflective Cognitive Modes. Mobile Computing in Education, 46-49 (2009)

6. Tano, Kodera, Nakashima, Kawano, Nakanish, Hamagishi, Inoue, Watanabe, Okamoto, Kawagoe, Kaneko, Hotta, Tatsuoka: Godzilla: Seamless 2D and 3D Sketch Environment for Reflective and Creative Design Work. In: INTERACT 2003, pp. 131-138 (2003)

7. Tano, S., Komatsu, Y., Iwata, M.: Extended Godzilla: Free-form 3D-object Design by Sketching and Modifying Seven Primitives at Single 2D-3D Seamless Display. In: Masoodian, M., Jones, S., Rogers, B. (eds.) APCHI 2004. LNCS, vol. 3101, pp. 471-480. Springer, Heidelberg (2004)

8. Tano, Sugimoto: Natural Hand Writing in Unstable 3D space with Artificial Surface. In: CHI 2001, pp. 353-354 (2001)

9. Tano, S., Matsumoto, T., Iwata, M.: Quantitative Analysis of Human Behavior and Implied User Interface in 3D Sketching. In: Masoodian, M., Jones, S., Rogers, B. (eds.) APCHI 2004. LNCS, vol. 3101, pp. 481-490. Springer, Heidelberg (2004)

10. Wayne, et al.: Interactive Augmented Reality Techniques for Construction at a Distance of 3D Geometry. In: Eurographics 2003, pp. 19-28 (2003)

11. Steven, et al.: Surface Drawing: Creating Organic 3D Shapes with the Hand and Tangible Tools. In: CHI 2001, pp. 261-268 (2001)

12. Gerols, et al.: Free Drawer: A Free-Form Sketching System on the Responsive Workbench. In: VRST 2001, pp. 167-174 (2001)

13. Daniel, et al.: A Fully Immersive 3D Artistic Medium and Interactive Experience. In: Proceedings 2001 ACM Symposium on Interactive 3D Graphics, pp. 85-93 (2001)

14. Tovi, et al.: Creating Principal 3D Curves with Digital Tape Drawing. In: CHI 2002, pp. 121-128 (2002)

15. Shin, H., Igarashi, T.: Magic canvas: interactive design of a 3-D scene prototype from freehand sketches. In: GI 2007, pp. 63-70 (2007)

16. Olsen, L., Samavati, F.F.: Stroke extraction and classification for mesh inflation. In: Proc. of SBIM 2010, pp. 9-16 (2010)

17. Perkunder, K., Israel, J.H., Alexa, M.: Shape modeling with sketched feature lines in immersive 3D environments. In: Proc. of SBIM 2010, pp. 127-134 (2010)

18. Tano, S., Yamamoto, M., Dzulkhiflee, M., Ichino, J., Hashiyama, T., Iwata, M.: Three Design Principles Learned through Developing a Series of 3D Sketch Systems: "Memory Capacity", "Cognitive Mode", and "Life-size and Operability". In: IEEE SMC 2012, pp. 880-887 (2012) 